Quim. Nova, Vol. 35, No. 10, 1940-1944, 2012

\title{
HIDROFORMILAÇÃO DO OLEATO DE METILA CATALISADA POR COMPLEXOS DE RÓDIO
}

\author{
Ana Nery Furlan Mendes \\ Departamento de Ciências Naturais, Centro Universitário Norte do Espírito Santo, Universidade Federal do Espírito Santo, Rod. \\ BR101 norte, km 60, s/n, 29900-000 São Mateus - ES, Brasil \\ Ricardo Gomes da Rosa e José Ribeiro Gregório* \\ Instituto de Química, Universidade Federal do Rio Grande do Sul, Av. Bento Gonçalves, 9500, 91501-970 Porto Alegre - RS, Brasil \\ Recebido em 18/1/12; aceito em 20/6/12; publicado na web em 31/8/12

\begin{abstract}
HYDROFORMYLATION OF METHYL OLEATE CATALYZED BY RHODIUM COMPLEXES. In this work, we describe the hydroformylation of methyl oleate catalyzed by several rhodium complexes. Parameters including total pressure, phosphorous/ rhodium and $\mathrm{CO} / \mathrm{H}_{2}$ ratio, temperature and phosphorous ligands were scanned. Total conversion of the starting double bonds was achieved while maintaining excellent selectivity in aldehydes.
\end{abstract}

Keywords: methyl oleate; hydroformylation; rhodium.

\section{INTRODUÇÃO}

Devido a fatores ambientais, muitos cientistas estão utilizando produtos obtidos de fontes renováveis, como óleos vegetais e seus ésteres, como substrato em diferentes processos. ${ }^{1}$ Dentre estas reações, a hidroformilação é uma das mais utilizadas, pois este processo pode ser realizado na presença de grupos funcionais como ésteres, amidas e nitrilas..$^{1-3} \mathrm{~A}$ economia de átomos desta reação é de $100 \%$, pois todos os átomos dos reagentes estão presentes no produto final, o que resulta em uma produção mínima de resíduos químicos. ${ }^{4} \mathrm{Uma}$ vez que a função aldeído é introduzida na cadeia de um éster graxo insaturado, como o oleato de metila, através da hidroformilação, tem-se um excelente material de partida para a formação de numerosos compostos, com aplicação em diversas áreas, como lubrificantes, plastificantes, tintas e formação de uretanas. ${ }^{1}$

A hidroformilação de ésteres graxos insaturados tem sido realizada principalmente com precursores catalíticos de ródio. Frankel relatou um sistema altamente seletivo para a hidroformilação do oleato de metila em 9(10)-formilestearato, utilizando condições relativamente brandas: $\mathrm{T}=95-110{ }^{\circ} \mathrm{C}, \mathrm{P}=34-140$ bar $\left(\mathrm{CO} / \mathrm{H}_{2}=1: 1\right)$, com e sem solvente. $\mathrm{O}$ catalisador de ródio ( $5 \% \mathrm{Rh}$ suportado em $\mathrm{C}, \mathrm{CaCO}_{3}$ ou $\mathrm{Al}_{2} \mathrm{O}_{3}$ ) na presença de trifenilfosfina e sob as condições da reação produziu conversões de $90-99 \%$ em produto hidroformilado. ${ }^{5} \mathrm{O}$ mesmo autor relatou a hidroformilação do linoleato e do linolenato de metila com catalisador de ródio (5\% $\mathrm{Rh}$ suportado em $\mathrm{Al}_{2} \mathrm{O}_{3}$ ) na presença de trifenilfosfina. ${ }^{6}$ A reação produziu 1,4-diformil ésteres como produtos majoritários e 1,3-diformil ésteres como produtos minoritários. Monoformil ésteres insaturados e saturados também foram produzidos para ambos os substratos, bem como triformil ésteres para o linolenato de metila.

A hidroformilação do oleato de metila $(\mathrm{OM})$ foi realizada por Friedrich, utilizando um catalisador de ródio suportado em alumina (5\% de Rh) e trifenilfosfito como ligante. ${ }^{7}$ Sob as condições da reação $\left(120{ }^{\circ} \mathrm{C}, 15\right.$ bar $\left(\mathrm{CO} / \mathrm{H}_{2}=1: 1\right)$, substrato/Rh = 100) o autor obteve $99 \%$ de conversão e $92 \%$ de seletividade em aldeído.

Muilwijk e colaboradores relataram uma série de experimentos realizados com o oleato de metila puro e com o de grau técnico, utilizando um catalisador de ródio modificado com um fosfito volumoso, o tris(2-tert-butil-4-metilfenil)fosfito. ${ }^{1}$ Sob condições de reação tais como $\mathrm{OM} / \mathrm{Rh}=910, \mathrm{~T}=80-100{ }^{\circ} \mathrm{C}, \mathrm{P}=20 \mathrm{bar}, \mathrm{CO} / \mathrm{H}_{2}=$

*e-mail: jrg@iq.ufrgs.br
1:1 e tolueno como solvente, $95 \%$ de conversão foi observada em 3 $\mathrm{h}$ de reação. Os autores também observaram a rápida isomerização do substrato formando a olefina com uma configuração trans, que reage mais lentamente do que o cis oleato original. $\mathrm{O}$ uso do oleato de metila grau técnico (contendo 14\% de linoleato de metila) resultou em baixas taxas de reação, pois, segundo os autores, dienos formam um intermediário $\pi$-alílico estável com o metal. Por isso, condições mais drásticas foram requeridas para se obter altas taxas de conversão e seletividade para esse substrato $\left(\mathrm{OM} / \mathrm{Rh}=910, \mathrm{~T}=120^{\circ} \mathrm{C}\right.$, $\mathrm{P}=50-80$ bar, $\left.\mathrm{CO} / \mathrm{H}_{2}=1: 1-1: 6\right)$. Muitos isômeros foram também formados durante a reação. A hidroformilação destes isômeros resultou em uma complexa mistura de produtos, consistindo principalmente de formilestearatos, formiloleatos e diformilestearatos de metila.

Behr relatou que os ésteres de ácidos graxos eram isomerizados a olefinas terminais no mesmo sistema que posteriormente conduziria à hidroformilação, obtendo de 26 a $34 \%$ de aldeídos lineares. ${ }^{8}$

Com base nos trabalhos publicados na literatura, a hidroformilação de ésteres graxos insaturados tem sido realizada utilizando-se principalmente precursores de ródio, como o $\left[\mathrm{Rh}(\mathrm{CO})_{2}(\mathrm{acac})\right]$. Segundo a literatura, o complexo $\left[\mathrm{RhH}(\mathrm{CO})\left(\mathrm{PPh}_{3}\right)_{3}\right]$ é um dos precursores mais ativos e estudados para a conversão de $\alpha$-olefinas em aldeídos. ${ }^{9-12}$ No entanto, nenhum estudo sistemático com este complexo foi realizado para a hidroformilação de olefinas internas, tais como os ésteres de ácidos graxos. Desta maneira, decidiu-se estudar a hidroformilação do oleato de metila utilizando o complexo $\left[\mathrm{RhH}(\mathrm{CO})\left(\mathrm{PPh}_{3}\right)_{3}\right]$, comparando os resultados com os obtidos empregando os seguintes precursores: $\left[\mathrm{RhCl}_{3} \cdot 3 \mathrm{H}_{2} \mathrm{O}\right],\left[\mathrm{RhCl}(\mathrm{CO})\left(\mathrm{PPh}_{3}\right)_{3}\right],\left[\mathrm{Rh}\left(\mathrm{OCH}_{3}\right)(1,5-\mathrm{COD})\right]_{2}$ e o $\left[\mathrm{Rh}(\mathrm{CO})_{2}(\mathrm{acac})\right]$. Foram também realizados testes preliminares utilizando-se os ligantes PEGPHOT e PEGPHOP, ${ }^{13}$ que podem diminuir a lixiviação já observada do complexo de ródio em meio bifásico, visando uma eventual reciclagem do sistema catalítico. ${ }^{14}$

\section{PARTE EXPERIMENTAL}

\section{Reagentes}

O oleato de metila (ACROS Organics, grau técnico, contendo $7,5 \%$ de linoleato de metila) foi usado após passagem por uma coluna de alumina para remover impurezas, como os peróxidos. Trifenilfosfina $\left(\mathrm{PPh}_{3}\right.$, Alpha, 99\%), trifenilfosfito $\left(\mathrm{P}(\mathrm{OPh})_{3}\right.$, Aldrich, 97\%) e $\left[\mathrm{RhCl}_{3} \cdot 3 \mathrm{H}_{2} \mathrm{O}\right]$ (Aldrich) foram utilizados sem 
prévia purificação. Os complexos $\left[\mathrm{RhH}(\mathrm{CO})\left(\mathrm{PPh}_{3}\right)_{3}\right]$, $[\mathrm{RhCl}(\mathrm{CO})$ $\left.\left(\mathrm{PPh}_{3}\right)_{3}\right],\left[\mathrm{Rh}\left(\mathrm{OCH}_{3}\right)(1,5-\mathrm{COD})\right]_{2}$ e $\left[\mathrm{Rh}(\mathrm{CO})_{2}(\mathrm{acac})\right]$ e os ligantes tri-(4-trietilenoglicolmonometiléterfenil)fosfito (PEGPHOT) e tri-(4-trietilenoglicolmonometiléterfenil)fosfina (PEGPHOP) foram preparados conforme descrito na literatura. ${ }^{13,15}$ Os gases $\mathrm{H}_{2}$ (AGA, 99,999\%) e CO (Air Products, 99,5\%) foram usados sem purificação.

\section{Métodos instrumentais de análise}

As análises de Ressonância Magnética Nuclear (RMN) foram realizadas em um equipamento Varian VXR-200 (RMN- ${ }^{-} \mathrm{H}$ a $200 \mathrm{MHz}$ e $\mathrm{RMN}-{ }^{13} \mathrm{C}$ a $50 \mathrm{MHz}$ ). As amostras foram preparadas em $\mathrm{CDCl}_{3}$, utilizando-se como referência o tetrametilsilano (TMS). As análises por espectroscopia no infravermelho (IV) foram realizadas em um espectrofotômetro no infravermelho com transformada de Fourier Mattson modelo 3020. As amostras foram preparadas na forma de filme sobre um cristal de $\mathrm{KBr}$. O espectro foi obtido por intermédio de medidas de transmitância, na faixa de 4000 a $500 \mathrm{~cm}^{-1}$, com 40 varreduras e resolução de $4 \mathrm{~cm}^{-1}$.

\section{Cálculos}

Através da análise dos sinais de hidrogênio presentes nos espectros de $\mathrm{RMN}-{ }^{1} \mathrm{H}$ do oleato de metila e das amostras hidroformiladas foram determinados o número de ligações duplas, a conversão e a seletividade das reações.

\section{Cálculo do número de ligações duplas para o oleato de metila (NDOM)}

Inicialmente, determinou-se o fator de normalização (FN), que representa a área correspondente a um hidrogênio. O sinal escolhido como padrão para cálculo do FN foi o do grupo metoxila. Este sinal foi escolhido, pois não varia com a modificação da cadeia carbônica. $\mathrm{O}$ fator $\mathrm{FN}$ foi calculado para cada espectro obtido. As atribuições dos sinais dos hidrogênios nos espectros de $\mathrm{RMN}^{-1} \mathrm{H}$ foram realizadas com base na literatura. ${ }^{16}$

$$
N D=\frac{(A / 2)}{F N}
$$

onde A é a área dos sinais dos hidrogênios das ligações duplas (Figura 1); $\mathrm{FN}=\mathrm{B} / 3$ (1/3 da área da metoxila) para o oleato de metila.

O oleato de metila grau técnico utilizado, apresenta em sua composição aproximadamente $7 \%$ de linoleato de metila.

Aplicando a Equação 1 para a amostra de OM utilizada nas reações de hidroformilação encontrou-se um valor de NDOM igual a 1,12.

Cálculo da conversão da reação de hidroformilação

$$
\text { Conversão }(\%)=\frac{N D_{i}-N D_{f}}{N D_{i}} \times 100
$$

onde NDi é o número de ligações duplas na amostra de partida; $\mathrm{ND}_{\mathrm{f}}$ é o número de ligações duplas que não foram convertidas em produtos, o qual é calculado da mesma forma que o anterior, considerando-se as áreas do espectro da amostra hidroformilada.

Cálculo da seletividade em aldeído (\%)

$$
\text { Aldeído }(\%)=\frac{J / F N}{N D_{i}-N D_{f}} \times 100
$$

onde $J$ é a área do sinal do hidrogênio de aldeído (9,5ppm).

$$
\begin{aligned}
& \mathrm{H}_{3} \mathrm{C}^{\mathrm{G}}-\left(\mathrm{CH}_{2}{ }^{\mathrm{F}}\right)_{6}-\mathrm{CH}_{2}{ }^{\mathrm{D}}-\mathrm{CH}^{\mathrm{A}}=\mathrm{CH}^{\mathrm{A}}-\mathrm{CH}_{2}{ }^{\mathrm{D}}-\left(\mathrm{CH}_{2}{ }^{\mathrm{F}}\right)_{4}-\mathrm{CH}_{2}{ }^{\mathrm{E}}-\mathrm{CH}_{2}{ }^{\mathrm{C}}-\mathrm{C} \\
& \mathrm{OCH}_{3}{ }^{\mathrm{B}}
\end{aligned}
$$

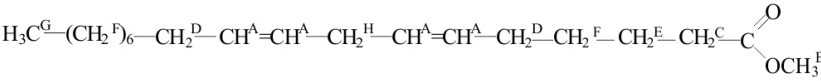

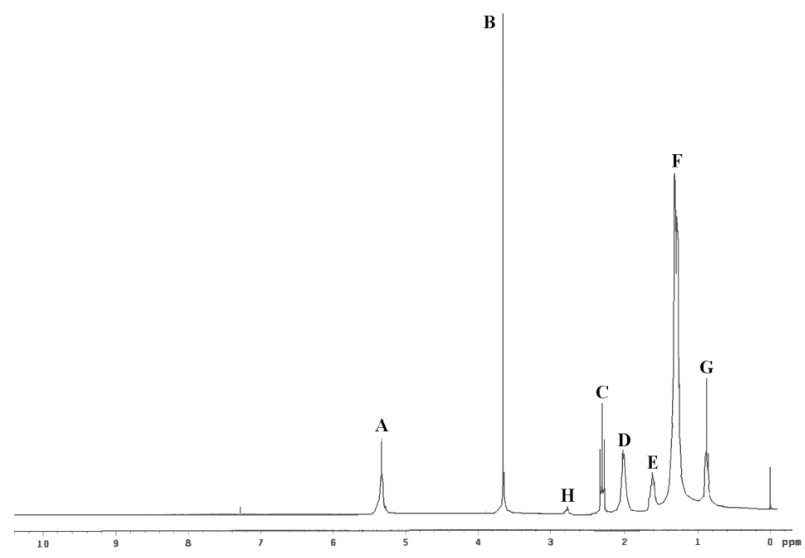

Figura 1. Espectro de $R M N{ }^{1}{ }^{-H}$ do oleato de metila grau técnico de partida

\section{Reações de hidroformilação}

As reações de hidroformilação do oleato de metila utilizado neste trabalho foram realizadas em um reator de aço inoxidável com $100 \mathrm{~mL}$ de capacidade interna, utilizando-se agitação magnética e na ausência de solvente. Em uma reação típica, o substrato, o precursor catalítico e o ligante foram transferidos para o reator, o qual foi selado e purgado três vezes com hidrogênio. Em seguida, o reator foi pressurizado com $\mathrm{H}_{2}$ e $\mathrm{CO}$ na proporção e pressão desejadas. $\mathrm{O}$ reator foi aquecido utilizando-se um banho de óleo de silicone e o início do tempo reacional contado a partir do aquecimento da mistura na temperatura do experimento, medida com o auxílio de um termopar adaptado ao reator.

As reações catalíticas foram finalizadas pelo resfriamento do reator à temperatura ambiente e posterior despressurização. Ao final de todos os experimentos, uma alíquota foi retirada para análise de RMN e IV e o restante da amostra foi passado em coluna de sílica-gel (utilizando-se diclorometano como eluente) e seco sob vácuo.

\section{RESULTADOS E DISCUSSÃO}

Os resultados iniciais das reações de hidroformilação do oleato de metila com o precursor $\left[\mathrm{RhH}(\mathrm{CO})\left(\mathrm{PPh}_{3}\right)_{3}\right]$ estão apresentados na Tabela 1, onde os valores são uma média de, no mínimo, duas reações.

Tabela 1. Resultados da hidroformilação do OM

\begin{tabular}{cccccc}
\hline Reação $^{(\mathrm{a})}$ & $\mathrm{T}\left({ }^{\circ} \mathrm{C}\right)$ & $\mathrm{t}(\mathrm{h})$ & $\begin{array}{c}\text { Conversão } \\
(\%)^{(\mathrm{b})}\end{array}$ & $\begin{array}{c}\text { Aldeído } \\
(\%)^{(\mathrm{b})}\end{array}$ & $\begin{array}{c}\text { Rendimento } \\
(\%)^{(\mathrm{c})}\end{array}$ \\
\hline 1 & 80 & 24 & $30 \pm 7$ & $50 \pm 15$ & $16 \pm 8$ \\
2 & 100 & 24 & $63 \pm 1$ & $94 \pm 1$ & $59 \pm 1$ \\
3 & 100 & 48 & $90 \pm 1$ & $80 \pm 15$ & $72 \pm 14$ \\
4 & 120 & 24 & $60 \pm 3$ & $64 \pm 22$ & $39 \pm 15$ \\
\hline
\end{tabular}

(a) $0,0238 \mathrm{mmol}\left[\mathrm{RhH}(\mathrm{CO})\left(\mathrm{PPh}_{3}\right)_{3}\right] ; 17,7 \mathrm{mmol}$ ligações duplas; $20 \mathrm{bar}(\mathrm{CO} /$ $\left.\mathrm{H}_{2}=1: 1\right) .{ }^{\left({ }^{b}\right)}$ Calculado por RMN- ${ }^{1} \mathrm{H}$. ${ }^{(\mathrm{c})}$ Conversão $\mathrm{x}$ seletividade

Analisando a Tabela 1 , verifica-se que a temperatura de $100{ }^{\circ} \mathrm{C}$ é melhor que a de $80^{\circ} \mathrm{C}$, pois forneceu maiores resultados de conversão 
e seletividade, sob as mesmas condições reacionais. Este fato está de acordo com a literatura, onde a reação de hidroformilação de ésteres graxos é conduzida a temperaturas entre 100 e $110{ }^{\circ} \mathrm{C}$, para se ter altas taxas de conversão. ${ }^{5}$

Comparando-se as reações 2 e 3, observa-se que há um aumento na conversão após um aumento do tempo reacional. Isto indica que o sistema catalítico se mantém ativo mesmo por longos períodos. No entanto, a seletividade do sistema apresenta grandes variações entre as repetições. Quando a reação 2 foi repetida aumentando-se a temperatura de $100{ }^{\circ} \mathrm{C}$ para $120^{\circ} \mathrm{C}$ (reação 4), baixas conversões foram alcançadas. Isto provavelmente se deve à decomposição térmica do catalisador, uma vez que este apresentava uma coloração escura ao final da reação, o que não acontecia nas reações anteriores. Este fato também foi observado na literatura para a hidroformilação do 1-hexeno, utilizando-se o complexo $\left[\mathrm{RhH}(\mathrm{CO})\left(\mathrm{PPh}_{3}\right)_{3}\right] \cdot{ }^{12} \mathrm{Os}$ autores concluíram que nas reações realizadas com temperaturas superiores a $100{ }^{\circ} \mathrm{C}$, ocorre uma desativação do sistema, com a formação de um composto com coloração marrom-escuro. Este composto foi classificado como sendo uma espécie inativa para a reação, devido à ortometalação do anel do ligante fosfina.

Embora as reações sob 20 bar de pressão total forneçam resultados satisfatórios, estes são de difícil reprodutibilidade, provavelmente devido à diferença de solubilidade dos gases no meio reacional ou à modificação da cinética da reação causada pelo consumo dos gases. Para minimizar estes problemas, optou-se por trabalhar com uma pressão total mais elevada (40 bar) com o intuito de realizar-se uma saturação dos gases no sistema reacional e, deste modo, diminuir os problemas difusionais. Os resultados obtidos das reações realizadas sob 40 bar estão apresentados na Tabela 2 (os valores são uma média de, no mínimo, duas reações).

Tabela 2. Resultados da hidroformilação do oleato de metila sob 40 bar de pressão

\begin{tabular}{ccccc}
\hline Reação $^{(\mathrm{a})}$ & $\begin{array}{c}\mathrm{t} \\
(\mathrm{h})\end{array}$ & $\begin{array}{c}\text { Conversão } \\
(\%)^{(\mathrm{b})}\end{array}$ & $\begin{array}{c}\text { Aldeído } \\
(\%)^{(\mathrm{b})}\end{array}$ & $\begin{array}{c}\text { Rendimento } \\
(\%)^{(\mathrm{c})}\end{array}$ \\
\hline 5 & 24 & $100 \pm 0$ & $55 \pm 3$ & $55 \pm 3$ \\
6 & 14 & $100 \pm 0$ & $62 \pm 2$ & $62 \pm 2$ \\
7 & 9 & $100 \pm 0$ & $59 \pm 3$ & $59 \pm 3$ \\
8 & 6 & $70 \pm 3$ & $80 \pm 2$ & $56 \pm 4$ \\
$9^{(\mathrm{d})}$ & 6 & $100 \pm 0$ & $48 \pm 5$ & $48 \pm 5$ \\
$10^{(\mathrm{e})}$ & 6 & $87 \pm 5$ & $46 \pm 5$ & $40 \pm 7$ \\
$11^{(\mathrm{d}, \mathrm{e})}$ & 6 & $100 \pm 0$ & $87 \pm 3$ & $87 \pm 3$ \\
$12^{(\mathrm{f})}$ & 6 & $100 \pm 0$ & $59 \pm 5$ & $59 \pm 5$ \\
$13^{(\mathrm{d}, \mathrm{e})}$ & 4 & $100 \pm 0$ & $87 \pm 2$ & $87 \pm 2$ \\
$14^{(\mathrm{de}, \mathrm{g}, \mathrm{g}}$ & 4 & $100 \pm 0$ & $60 \pm 2$ & $60 \pm 2$ \\
\hline
\end{tabular}

(a) $0,0238 \mathrm{mmol}\left[\mathrm{RhH}(\mathrm{CO})\left(\mathrm{PPh}_{3}\right)_{3}\right] ; 17,7 \mathrm{mmol}$ ligações duplas; $40 \mathrm{bar}(\mathrm{CO} /$ $\left.\mathrm{H}_{2}=1: 1\right) ; 100{ }^{\circ} \mathrm{C} .{ }^{(b)}$ Calculado por RMN- ${ }^{1} \mathrm{H} .{ }^{(\mathrm{c})}$ Conversão x seletividade. ${ }^{(\mathrm{d})}$ Reação realizada adicionando-se um excesso de 7 vezes de $\mathrm{PPh}_{3}\left(\mathrm{PPh}_{3} / \mathrm{Rh}\right.$ $=10)$. ${ }^{(\mathrm{e})}$ Reação realizada com um excesso de $\mathrm{CO}\left(\mathrm{CO} / \mathrm{H}_{2}=2: 1\right)$. ${ }^{(\mathrm{f})}$ Reação realizada adicionando-se um excesso de 4 vezes de $\mathrm{PPh}_{3}\left(\mathrm{PPh}_{3} / \mathrm{Rh}=7\right)$. ${ }^{\text {(g) }}$ Reação realizada com $5 \mathrm{~mL}$ de hexano

Analisando-se os resultados da Tabela 2, observa-se que, graças à saturação da solução reacional com $\mathrm{CO} / \mathrm{H}_{2}$, pode-se reduzir o tempo de reação para um valor menor ou igual a $9 \mathrm{~h}$.

\section{Efeito do excesso de trifenilfosfina}

Como a reação 8, embora incompleta, foi a que apresentou o melhor valor de seletividade, resolveu-se realizar esta mesma reação com excesso de $\mathrm{PPh}_{3}$, a fim de aumentar o tempo de vida útil do catalisador. O resultado obtido pode ser visto na reação 9 , onde se observa uma melhora na conversão do sistema. Na literatura há alguns trabalhos em que foi observado que um excesso de fosfina promove uma melhora na conversão, quando comparada com a mesma reação sem excesso de fosfina. ${ }^{5,12,13}$ No entanto, nenhum trabalho forneceu evidências experimentais sobre o mecanismo pelo qual este excesso de fosfina atua. Alguns autores sugerem que a presença de ligantes fosforados suprima espécies que levem à isomerização e/ou à hidrogenação do substrato. ${ }^{17-19}$

\section{Influência da proporção de gás de síntese}

O efeito da proporção de $\mathrm{CO}$ e $\mathrm{H}_{2}$ sobre o desempenho do sistema catalítico foi estudado comparando-se as reações 8 e 10 . Observouse que a maior quantidade de $\mathrm{CO}$ no meio reacional promove uma melhora na conversão da reação. Além disso, a seletividade é comparável com o resultado obtido na reação onde um excesso de fosfina foi utilizado (reação 9).

Como as reações 9 e 10 foram as que apresentaram os melhores valores de conversão, decidiu-se realizar uma reação utilizando um excesso de trifenilfosfina e de $\mathrm{CO}$, com a intenção de melhorar a seletividade (reação 11). O resultado da reação 11 foi igual ao da reação 9 em termos de conversão, mas a seletividade do sistema teve um aumento de aproximadamente $40 \%$. Na literatura há alguns trabalhos sobre a hidroformilação de ésteres graxos, em que um aumento na seletividade do sistema foi atingido quando as reações foram realizadas com um excesso de fosfina. Os autores atribuem este resultado à diminuição da taxa de isomerização e, consequentemente, um aumento na taxa de hidroformilação. ${ }^{1,17-19}$ Frankel e colaboradores verificaram que a extensão da migração da ligação dupla é inversamente proporcional à concentração de fosfina no meio reacional. ${ }^{5}$ Muilwijk e colaboradores verificaram que o excesso de fosfina inibe a isomerização posicional e, também, a isomerização geométrica da ligação dupla. ${ }^{1}$ Trzeciak observou, na reação de hidroformilação do 1,5-hexadieno, que a isomerização da ligação dupla é minimizada quando a reação é realizada com um excesso de $\mathrm{CO}$ sobre $\mathrm{H}_{2} \cdot{ }^{20} \mathrm{~A}$ partir disto, conclui-se que a melhora na seletividade da reação 11 deve-se ao somatório dos efeitos do excesso de fosfina e de $\mathrm{CO}$, que minimizaram as reações de isomerização e de hidrogenação da ligação dupla do oleato de metila e favoreceram a hidroformilação do substrato.

Com a intenção de verificar se uma menor razão fosfina/ródio produz os mesmos resultados obtidos na reação 11 , realizou-se a reação 12 com uma proporção $\mathrm{PPh}_{3}: \mathrm{Rh}$ igual a 7:1 (adição de um excesso de 4 vezes de trifenilfosfina). Como resultado obteve-se $100 \%$ de conversão, mas uma seletividade em aldeídos menor, o que sugere que a proporção ideal de $\mathrm{PPh}_{3}: \mathrm{Rh}$ seja igual a 10:1, para as condições reacionais empregadas.

Realizando-se a reação 11 com um tempo reacional menor (reação 13), observa-se que com 4 h obtém-se os mesmos resultados.

$\mathrm{Na}$ reação 14, as mesmas condições reacionais da reação 13 foram utilizadas, porém adicionando-se ao reator $5 \mathrm{~mL}$ de hexano seco. Nesta reação obteve-se $100 \%$ de conversão, mas uma menor seletividade em aldeídos, quando comparada com a reação 13. Este aumento na porcentagem de hidrogenação pode ser atribuído à melhor solubilidade do hidrogênio neste meio.

Com base nos resultados obtidos na hidroformilação do oleato de metila utilizando-se o complexo $\left[\mathrm{RhH}(\mathrm{CO})\left(\mathrm{PPh}_{3}\right)_{3}\right]$, pode-se definir as seguintes condições reacionais como sendo ótimas para a hidroformilação de ésteres graxos insaturados: temperatura $100{ }^{\circ} \mathrm{C}$; tempo $4 \mathrm{~h}$; pressão total 40 bar; proporção $\mathrm{CO}: \mathrm{H}_{2}$ de 2:1; proporção $\mathrm{PPh}_{3}: \mathrm{Rh}$ de 10:1.

\section{Hidroformilação com diferentes precursores de ródio}

Utilizando-se as condições otimizadas obtidas para o complexo 
Tabela 3. Hidroformilação do oleato de metila com diferentes precursores catalíticos

\begin{tabular}{|c|c|c|c|c|c|c|}
\hline Reação ${ }^{(a)}$ & complexo & ligante & $\mathrm{t}(\mathrm{h})$ & $\mathrm{C}(\%)^{(\mathrm{b})}$ & $\mathrm{S}(\%)^{(\mathrm{b})}$ & $\mathrm{R}(\%)^{(\mathrm{c})}$ \\
\hline 15 & {$\left[\mathrm{RhCl}_{3} \cdot 3 \mathrm{H}_{2} \mathrm{O}\right]$} & $\mathrm{PPh}_{3}$ & 4 & 34 & 28 & 10 \\
\hline 16 & {$\left[\mathrm{RhCl}(\mathrm{CO})\left(\mathrm{PPh}_{3}\right)_{3}\right]$} & $\mathrm{PPh}_{3}$ & 4 & 100 & 75 & 75 \\
\hline 17 & $\begin{array}{c}{[\mathrm{Rh}(\mathrm{OMe})(1,5-} \\
\mathrm{COD})]_{2}\end{array}$ & $\mathrm{PPh}_{3}$ & 4 & 46 & 66 & 30 \\
\hline 18 & {$\left[\mathrm{Rh}(\mathrm{CO})_{2}(\mathrm{acac})\right]$} & $\mathrm{PPh}_{3}$ & 4 & 47 & 67 & 31 \\
\hline 19 & {$\left[\mathrm{Rh}(\mathrm{CO})_{2}(\mathrm{acac})\right]$} & $\mathrm{PPh}_{3}$ & 20 & 90 & 90 & 81 \\
\hline 20 & {$\left[\mathrm{Rh}(\mathrm{CO})_{2}(\mathrm{acac})\right]$} & $\mathrm{P}(\mathrm{OPh})_{3}$ & 6 & 49 & 62 & 30 \\
\hline 21 & {$\left[\mathrm{Rh}(\mathrm{CO})_{2}(\mathrm{acac})\right]$} & $\mathrm{P}(\mathrm{OPh})_{3}$ & 9 & 70 & 67 & 47 \\
\hline 22 & {$\left[\mathrm{Rh}(\mathrm{CO})_{2}(\mathrm{acac})\right]$} & $\mathrm{P}(\mathrm{OPh})_{3}$ & 20 & 100 & 80 & 80 \\
\hline 23 & {$\left[\mathrm{Rh}(\mathrm{CO})_{2}(\mathrm{acac})\right]$} & PEGPHOT & 6 & 29 & 67 & 19 \\
\hline 24 & {$\left[\mathrm{Rh}(\mathrm{CO})_{2}(\mathrm{acac})\right]$} & PEGPHOT & 20 & 100 & 71 & 71 \\
\hline 25 & {$\left[\mathrm{Rh}(\mathrm{CO})_{2}(\mathrm{acac})\right]$} & PEGPHOP & 20 & 100 & 78 & 78 \\
\hline
\end{tabular}

${ }^{\text {(a) }} 0,0238$ mmol de precursor catalítico; 17,7 mmol ligações duplas; 40 bar; $100{ }^{\circ} \mathrm{C} ; \mathrm{CO} / \mathrm{H}_{2}=2: 1, \mathrm{~L} / \mathrm{Rh}=10: 1$. ${ }^{\text {b) }} \mathrm{Calculado}$ por RMN- ${ }^{1} \mathrm{H}$. ${ }^{\text {(c) }} \mathrm{Conversão} \mathrm{x}$ seletividade

$\left[\mathrm{RhH}(\mathrm{CO})\left(\mathrm{PPh}_{3}\right)_{3}\right]$, verificou-se o efeito que outros precursores à base de ródio possuem na hidroformilação do oleato de metila. Desta maneira, decidiu-se testar os complexos $\left[\mathrm{RhCl}_{3} \cdot 3 \mathrm{H}_{2} \mathrm{O}\right],[\mathrm{Rh}(\mathrm{OMe})$ $(1,5-\mathrm{COD})]_{2},\left[\mathrm{Rh}(\mathrm{CO})_{2}(\mathrm{acac})\right]$ e $\left[\mathrm{RhCl}(\mathrm{CO})\left(\mathrm{PPh}_{3}\right)_{3}\right]$, sendo que os resultados obtidos encontram-se descritos na Tabela 3.

Comparando os resultados obtidos nas reações 15 a 18 com aqueles obtidos para o precursor $\left[\mathrm{RhH}(\mathrm{CO})\left(\mathrm{PPh}_{3}\right)_{3}\right]$, nota-se que somente o complexo $\left[\mathrm{RhCl}(\mathrm{CO})\left(\mathrm{PPh}_{3}\right)_{3}\right]$ forneceu resultados semelhantes sob as mesmas condições reacionais. Sabe-se que na reação de hidroformilação com precursores de ródio, a espécie ativa que se forma na presença de $\mathrm{CO}, \mathrm{H}_{2}$ e ligante (L) é a $\mathrm{RhHCOL}_{2} \cdot{ }^{17} \mathrm{Os}$ complexos $\left[\mathrm{RhH}(\mathrm{CO})\left(\mathrm{PPh}_{3}\right)_{3}\right]$ e $\left[\mathrm{RhCl}(\mathrm{CO})\left(\mathrm{PPh}_{3}\right)_{3}\right]$ apresentam estruturas mais próximas da espécie cataliticamente ativa para a reação (quando $\mathrm{L}$ $=\mathrm{PPh}_{3}$ ), diminuindo-se assim o tempo necessário para a ativação do catalisador. Com isto, acredita-se que os demais precursores utilizados, por não apresentarem inicialmente estruturas semelhantes à da espécie cataliticamente ativa, necessitam de mais tempo para ativação nas condições reacionais empregadas. Isto fica claramente evidenciado analisando-se o resultado da reação 19 , realizada com um tempo reacional maior, obtendo-se um resultado bem próximo ao obtido com o complexo $\left[\mathrm{RhH}(\mathrm{CO})\left(\mathrm{PPh}_{3}\right)_{3}\right]$.

\section{Efeito de diferentes ligantes}

Para verificar o efeito que diferentes ligantes fosforados possuem sobre a hidroformilação do oleato de metila, decidiu-se utilizar como precursor catalítico o complexo $\left[\mathrm{Rh}(\mathrm{CO})_{2}(\mathrm{acac})\right]$. Além da trifenilfosfina, foram utilizados os ligantes trifenilfosfito, tri-(4-trietilenoglicolmonometiléterfenil)fosfito (PEGPHOT) e tri-(4-trietilenoglicolmonometiléterfenil)fosfina (PEGPHOP), sendo que os dois últimos são ligantes recentemente sintetizados pelo nosso grupo de pesquisa. Estes testes visaram a obtenção de informações quanto à viabilidade da reciclagem do precursor catalítico quando a reação for realizada utilizando-se polietilenoglicol como solvente. ${ }^{13}$ Os resultados obtidos estão apresentados na Tabela 3 (reações 2025). Pode-se observar que para as condições reacionais utilizadas, é necessário um maior tempo reacional para a ativação do catalisador. Nas reações realizadas com trifenilfosfito como ligante (reações 20-22) foram necessárias $20 \mathrm{~h}$ de reação para se obter os mesmos resultados encontrados para o precursor $\left[\mathrm{RhH}(\mathrm{CO})\left(\mathrm{PPh}_{3}\right)_{3}\right]$ com trifenilfosfina. Com o ligante PEGPHOT (reações 23-24), obtiveram-se os mesmos valores de conversão e seletividade do que para o ligante trifenilfosfito. Para o ligante PEGPHOP, com 20 h de reação, obteve-se conversão total das ligações duplas do oleato de metila e elevada seletividade em aldeídos.

\section{CONCLUSÕES}

A partir dos resultados obtidos conclui-se que o complexo $\left[\mathrm{RhH}(\mathrm{CO})\left(\mathrm{PPh}_{3}\right)_{3}\right]$ é um excelente catalisador para a hidroformilação de ésteres graxos insaturados de origem vegetal. Na hidroformilação do oleato de metila, observou-se que as reações são mais reprodutíveis quando são realizadas com 40 bar de pressão total, pois a 20 bar de pressão há sérios problemas difusionais. Além disso, o uso de um excesso de $\mathrm{PPh}_{3}$ sobre Rh (10:1) e de CO sobre $\mathrm{H}_{2}$ (2:1), simultaneamente, aumenta a conversão e a seletividade do sistema. $\mathrm{O}$ uso dos precursores $\left[\mathrm{RhCl}_{3} .3 \mathrm{H}_{2} \mathrm{O}\right],[\mathrm{Rh}(\mathrm{OMe})(1,5-\mathrm{COD})]_{2} \mathrm{e}\left[\mathrm{Rh}(\mathrm{CO})_{2}(\mathrm{acac})\right]$ com o ligante trifenilfosfina não produz os mesmos resultados obtidos para os complexos $\left[\mathrm{RhH}(\mathrm{CO})\left(\mathrm{PPh}_{3}\right)_{3}\right]$ e $\left[\mathrm{RhCl}(\mathrm{CO})\left(\mathrm{PPh}_{3}\right)_{3}\right]$, sob as mesmas condições reacionais. Provavelmente isto é devido às estruturas destes últimos serem mais próximas à da espécie cataliticamente ativa, necessitando um menor tempo para a ativação do catalisador. $\mathrm{O}$ uso de $\left[\mathrm{Rh}(\mathrm{CO})_{2}(\mathrm{acac})\right]$ como precursor catalítico, com diferentes ligantes fosforados, produz elevadas conversões e seletividades em aldeídos, porém em um tempo reacional maior do que aquele observado para o complexo $\left[\mathrm{RhH}(\mathrm{CO})\left(\mathrm{PPh}_{3}\right)_{3}\right]$. Não se observaram diferenças significativas entre os ligantes fosforados testados quando o tempo reacional foi de $20 \mathrm{~h}$.

\section{AGRADECIMENTOS}

Ao PRONEX/CNPq/FAPERGS-04/0887-0 e 10/00506, FAPERGS 04/1117.9, Spanish DGI (projeto CTQ200509187-C02-01) e ao CNPq (projetos 474050/2007-6, 473917/2008-4 e 310967/2009-0) pelo suporte financeiro, bem como à CAPES e ao CYTED (Projeto V9), por bolsas para A. N. F. Mendes durante seu estágio no laboratório do Prof. J. C. Bayón (Universitat Autònoma de Barcelona).

\section{REFERÊNCIAS}

1. Muilwijk, K. F.; Kamer, P. C. J.; van Leeuwen, P. W. N. M.; J. Am. Oil Chem. Soc. 1997, 74, 223.

2. Clarke, M. L.; Tetrahedron Lett. 2004, 45, 4043.

3. Cuny, G. D.; Buchwald, S. L.; J. Am. Chem. Soc. 1993, 115, 2066.

4. Trost, B. M.; Angew. Chem., Int. Ed. 1995, 34, 259. 
5. Frankel, E. N.; J. Am. Oil Chem. Soc. 1971, 48, 248.

6. Frankel, E. N.; Thomas, F. L.; Rowedder, W. K.; Ind. Eng. Chem. Prod. Res. Develop. 1973, 12, 47.

7. Friedrich, J. P.; Ind. Eng. Chem. Prod. Res. Develop. 1978, 17, 205.

8. Behr, A.; Obst, D.; Westfechtel, A.; Eur. J. Lipid. Sci. Technol. 2005, 107, 213.

9. Mukhopadhyay, K.; Mandale, A. B.; Chaudhari, R. V.; Chem. Mater. 2003, 15, 1766.

10. Bianchini, C.; Lee, H. M.; Meli, A.; Vizza, F.; Organometallics 2000, 19,849 .

11. Bhanage, B. M.; Divekar, S. S.; Deshpande, R. M.; Chaudhari, R. V.; J. Mol. Catal. A: Chem. 1997, 115, 247.

12. Deshpande, R. M.; Divekar, S. S.; Gholap, R. V.; Chaudhari, R. V.; J. Mol. Catal. 1991, 67, 333.

13. Gregório, J. R.; da Rosa, R. G.; Mendes, A. N. F.; Bayón, J. C.; Catal. Lett. 2011, 141, 977; Gregório, J. R.; da Rosa, R. G.; Mendes, A. N. F.;
Bayón, J. C.; Resumos da 34a Reunião Anual da Sociedade Brasileira de Química, Florianópolis, Brasil, 2011; Mendes, A. N. F.; Tese de Doutorado, Universidade Federal do Rio Grande do Sul, Brasil, 2007.

14. da Rosa, R. G.; Martinelli, L., da Silva L. H. M.; Loh, W.; Chem. Commun. 2000, 1, 33 .

15. Osborn, J. A.; Wilkinson, G.; Inorg. Synth. 1990, 28, 82.

16. Miyake, Y.; Yokomizo, K.; Matsuzaki, N.; J. Am. Oil Chem. Soc. 1998 , $75,1091$.

17. van Leeuwen, P. W. N. M.; Casey, C. P.; Whiteker, G. T. Em Rhodium Catalyzed Hydroformylation; van Leeuwen, P. W. N. M.; Claver, C., eds.; Springer: Dordrecht, 2000, chap. 4.

18. Barros, H.; Guimarães, C.; dos Santos, E. N.; Gusevskaya, E. V.; Catal. Commun. 2007, 8, 747 .

19. Barros, H. J. V.; da Silva, J. G.; Guimarães, C. C.; dos Santos, E. N.; Gusevskaya, E. V.; Organometallics 2008, 27, 4523.

20. Trzeciak, A. M.; Ziólkowski, J. J.; J. Organomet. Chem. 1994, 464, 107. 